\title{
Physiological effects of a pollutant gradient - summary
}

\author{
J. M. Capuzzo* \\ Department of Biology, Woods Hole Oceanographic Institution, Woods Hole, Massachusetts 02543, USA
}

The objective of the physiological studies at the GEEP Workshop was to assess the following responses to field and experimental pollutant gradients: (1) bioenergetic parameters, such as scope for growth, as an integrative measure of energy allocation to somatic and reproductive processes; (2) specific components of a bioenergetic budget, such as respiration and excretion; (3) larval viability from controlled spawnings; (4) lipid composition of digestive gland in relation to nutrient storage and turnover.

\section{SCOPE FOR GROWTH}

A marked decline in scope for growth (SFG) was observed in Mytilus edulis with increasing body burdens of polycyclic aromatic hydrocarbons (PAHs) and polychlorinated biphenyls (PCBs) in field samples, and of PAHs and copper in the mesocosms. Clearance $1=$ feeding) rate was the component of the energy budget that was primarily affected. Little difference in respiration rates, absorption efficiencies or excretion rates was evident with either field or mesocosm contaminants, with the exception of the high-dose mesocosm basin where the first two were significantly reduced. In both mesocosm and field studies, results indicated simple multiplicative (i.e. proportionally additive) effects of the different contaminants.

SFG was responsive not only to contaminant gradients but also to differences in seston (food ration) concentration, reflected in the lower growth potential of Mytilus edulis in the mesocosms than in the field. SFG has utility under both standardized conditions, where effects of pollutants are separated from other environmental factors such as seston concentration, and under in situ field conditions where the interaction of environmental factors and contaminants may be determined.

\footnotetext{
- This summary was written following discussions amongst all contributors to this section
}

The concept of SFG can, in theory, be applied to all animals. In practice, however, many species show cyclic or erratic feeding and metabolic activities which make estimation of physiological parameters difficult without relying on integrated measures over several days. For SFG to be a useful tool in monitoring biological effects of pollutants, individual physiological components must be monitored within $24 \mathrm{~h}$, before significant recovery from pollutant exposure can occur. The mussel meets this criterion because it maintains clearance and respiration rates at relatively constant levels; in addition it does not suffer 'handling stress'. In both field and mesocosm studies, SFG in Mytilus edulis proved to be an extremely sensitive indicator of physiological response to contaminant gradients and agreed well with both measures of biochemical and cellular processes and predictions of impacts based on bioaccumulation data.

In the field study with Littorina littorea, however, there was no clear relation between SFG measurements and the contaminant gradient, with periwinkles from one of the more impacted sites (4) exhibiting the highest readings and no differences being seen between the remaining sites. In the mesocosm experiment with $L$. littorea, bioenergetic responses failed to discriminate among the exposure conditions, with the exception of reduced respiration rates in the dosed basins, relative to the control. The lack of consistent trends in SFG of $L$. littorea in response to contaminants was the result of erratic feeding activity and unpredictable behavioural responses to fluctuations in food availability and quality. At present, its use as a monitoring tool in L. littorea is premature and both method development and a better understanding of bioenergetic processes in such gastropod molluscs are required.

\section{RESPIRATION AND EXCRETION}

A comparison of rates of respiration and nitrogen excretion, presented in the form of either nitrogen 
quotients (NQ) or oxygen: nitrogen ratios, showed no significant differences for Mytilus edulis or Littorina littorea, with the exception of values calculated for $M$. edulis in the high-dose mesocosm where ammonia excretion rates were significantly lower than for other treatments. In field populations nitrogen quotients calculated for $M$. edulis, and $\mathrm{O}: \mathrm{N}$ ratios for $L$. littore $\mathrm{A}_{1}$ were significantly different at Sites 2, 3 and 4 ; this indicated lower rates of protein catabolism. In the mesocosm experiment with the deposit-feeding bivalve Nucula tenuis, increased rates of protein catabolism were observed from low and medium-dose basins (there was no material available from high-dose exposures). These differences in physiological measurements coincided with water-column concentrations of diesel oil and copper in the basins but did not correlate with sediment concentrations. Interspecific differences in the sensitivity of protein catabolism and protein turnover in response to contaminant gradients warrant further exploration to assess their potential as monitoring tools.

Tissue respiration rates are of limited applicability as physiological measures since they reflect the metabolic demands of specific tissues to contaminant gradients and are not an integrated (i.e. 'whole animal') response. Their utility for monitoring will vary with the nature of the contamination and the sensitivity of the species. Gill respiration rates of Carcinus maenas were elevated at the 2 most contaminated field sites ( 3 and 4); these changes coincided with increased body burdens of PAHs and PCBs, but did not coincide directly with changes in gill tissue concentrations of copper (F. Thurberg pers. comm.). Similar measurements on gill preparations from crabs in the mesocosm experiment were insensitive to the contaminant gradient. Gill respiration rates of Mytilus edulis were insensitive to contaminant gradients in both field and mesocosm studies, and did not correlate with scope for growth measurements. Gill respiration rates of $M$. edulis did correlate with measurements of whole animal respiration but they are not an adequate estimate of whole animal energetics.

\section{LARVAL BIOASSAY}

The larval bioassay, using Mytilus edulis incubated in each of the mesocosm basins, showed a pattern of larval development similar to responses in scope for growth of adults. The relative agreement of a shortterm measure such as the bioassay with a measure that integrates responses over a longer time period is promising in selecting multiple techniques for pollution monitoring.

\section{LIPID COMPOSITION}

In the Langesundfjord, Norway, changes in lipid content and lipid:protein ratios of digestive glands of Mytilus edulis and Carcinus maenas reflected the contamination gradient. In the mesocosm experiment only $M$. edulis from the high-dose basin showed elevations in lipid content and lipid: protein ratios.

Analysis of lipid class composition for field populations of Mytilus edulis suggests decreased mobilization of triacylglycerols into phospholipid pools with potential consequences for membrane structure and function. Mytilus edulis from the mesocosm experiment did not show consistent trends among the various conditions, in part reflecting differences in reproductive condition and nutritional status. Mussels from the highdose basin, however, were distinctly different from other conditions, having significantly lower phospholipid content, suggesting membrane destruction, and marked increases in both the neutral lipid:polar lipid and triacylglycerol : phospholipid ratios. Changes in lipid class distributions of $M$. edulis from both field and mesocosm studies are consistent with histological and histopathological changes and correlate well with body burden data for tissue concentrations of PAHs and PCBs.

Observations on Carcinus maenas from the contaminated field sites suggest that alterations in the mobilization of triacylglycerols, sterol turnover, and tissue degeneration may have taken place. Responses to the contaminant gradient were consistent with changes in respiration rate and elevations in glutathione transferase activity. The responses were not consistent with $\mathrm{PAH}$ or PCB tissue concentrations. Crabs from the mesocosm experiment showed no evidence of alterations in lipid class distribution in spite of the clear trend in aromatic hydrocarbon tissue concentrations across the basins. This is consistent with other mesocosm studies of $C$. maenas, as no differences in either gill respiration rate or glutathione transferase activity were observed. In comparison with field populations of $C$. maenas, there was no evidence of metabolic turnover of aromatic hydrocarbons or of molting among any mesocosm crabs.

Differential responses of Mytilus edulis and Carcinus maenas are possibly the result of differences in metabolic capacity for detoxification and in trophic transfer. 